\title{
The surgical treatment of BRONJ: Parma Maxillofacial Surgery School experience
}

\author{
T Poli , S Ferrari, E Sesenna \\ From de Senectute: Age and Health Forum \\ Catanzaro, Italy. 5-7 December 2009
}

\section{Background}

The surgical treatment of patients affected by BRONJ (Bisphosphonate-related osteonecrosis of the jaw) represents one of the most debated issues in the international literature becouse the data reported to date are preliminary results of long-term studies.

In accordance with the guidelines proposed in 2007 by the American Association of Oral and Maxillofacial Surgeons position paper on bisphosphonate-Related Osteonecrosis of the Jaws " and confirmed by the Update 2009 of the same document, surgical treatment should be reserved only to cases of BRONJ in an advanced stage (Stage III) or in the presence of a well-defined bone sequestration. Unfortunately the proposed classification, even if very schematic and user-friendly, in our opinion is still too rigid and does not take into account the patient as a whole.

\section{Materials and methods}

At the University Hospital of Parma, a study group has been formed since 2005 for the treatment of patients with BRONJ, involving the various specialties involved in the management of this disease (dentists, maxillo facial surgeons, pathologists, medical oncologists)

To date $150 \mathrm{BRONJ}$ patients were enrolled and treated at the Section of Dentistry and Section of Maxillofacial Surgery.

\section{Results}

In our patients with BRONJ encouraging results have been achieved by surgical therapy performed early (stage I or II, with or without the use of lasers), bringing valuable clinical success over $80 \%$ of treated sites, compared with results obtained with medical therapy alone (no clinical nor symptomatic stable improvement for at least three months after treatment).

\section{Conclusions}

The decision whether to surgically treat the patient not in an advanced stage, but at the beginnings of necrosis (e.g., in presence of an asymptomatic mucosal dehiscence or of a fistulous intraoral tract) is justified essentially by two elements. The first is that if the bone involvement is still limited (stage I or II) the intervention will undoubtedly be less burdensome for the patient with a surgical operation under local anaesthesia and little post-operative hospitalization. The second is that the possibility of a tight closure of mucosal flaps creates an anatomical barrier to pathogenic microorganisms. In this way it would limit the risk of progression of infection with the related complications in terms of symptoms and functional impairment..

The School of Parma sustains that, without the possibility to reach healing as histological and radiological restitution ad integrum, it is necessary to adopt a classification of the level of clinical results obtained with the surgical procedure which considers the clinical symptomatology and objectivity, called Level Clinical Success.

Published: 19 May 2010

doi:10.1186/1471-2318-10-S1-A72

Cite this article as: Poli et al:: The surgical treatment of BRONJ: Parma Maxillofacial Surgery School experience. BMC Geriatrics 2010 10(Suppl 1):A72.
Maxillofacial Surgery Section, Head and Neck Department, University Hospital of Parma, Parma, Italy 